\title{
Cytokines reinstate NK cell-mediated cancer immunosurveillance
}

\author{
Laurence Zitvogel ${ }^{1,2,3,4}$ and Guido Kroemer ${ }^{1,5,6,7,8}$ \\ ${ }^{1}$ Custave Roussy Cancer Campus, Villejuif, France. ${ }^{2}$ INSERM U1015, Villejuif, France. ${ }^{3}$ Université Paris-Sud XI, Faculté de Médecine, Le Kremlin Bicêtre, France. ${ }^{4}$ Center of Clinical Investigations in Biotherapies \\ of Cancer (CICBT) 507, Villejuif, France. ${ }^{5}$ Université Paris Descartes, Sorbonne Paris Cité, Paris, France. ${ }^{6}$ Metabolomics and Cell Biology Platforms, Gustave Roussy, Villejuif, France. ${ }^{7}$ Equipe 11 labelisée par la \\ Ligue contre le Cancer, Centre de Recherche des Cordeliers, INSERM U1138, Paris, France. ${ }^{8}$ Pôle de Biologie, Hôpital Européen Ceorges Pompidou, AP-HP, Paris, France.
}

\begin{abstract}
In healthy individuals, cells that lose expression of MHC class I molecules are quickly targeted for elimination by NK lymphocytes. A paradox in cancer immunology is the observation that many tumor cells often have a drastic reduction of MHC class I molecules, yet these cells are not eliminated by NK cells, as they should be. In this issue of the $\mathrm{JCl}$, Ardolino et al. demonstrate that NK cells that infiltrate MHC class I-deficient tumors acquire an anergic state that can be reversed by particular combinations of exogenous cytokines. These results indicate that IL-12 plus IL-18 or a recombinant interleukin engineered to stimulate the IL-2 receptor $\beta / \gamma$ heterodimer (but not the IL-2 receptor $\alpha / \beta / \gamma$ complex) have the potential to be used clinically to reinstate immunosurveillance against MHC class I-deficient tumors.
\end{abstract}

\section{MHC class I and NK cell education}

NK cells are innate lymphocytes programmed to kill target cells and also secrete cytokines to modulate adaptive immunity (1). NK cells can exert their effector functions against infected and malignant target cells while maintaining self-tolerance. The threshold of NK cell responsiveness is tuned by microenvironmental cues, and adaptability of NK cells to their milieu is dictated by their "education" or "licensing" by host MHC class I molecules, which operate through interaction with NK cell inhibitory receptors (killer inhibitor receptors [KIRs] in humans, Ly49 molecules in mice, and CD94/NKG2A dimers in both species). Hence, "uneducated" or "unlicensed" NK cells generated in MHC class I-deficient mice are defective in their response to tumor cells or antibodies that engage activating $\mathrm{NK}$ receptors (2). The adoptive transfer of NK cells derived from MHC class Ideficient hosts into WT, MHC class Iexpressing recipients results in functional recovery and full-blown responsiveness of the NK cells. Conversely, NK cells from WT animals that are transferred into MHC class I-deficient hosts become hyporesponsive or anergic. Similarly, human NK cells that lack killer cell immunoglobulin-like receptor (KIR) display low killing and cytokine release capacities when encountering target cells (2). The most responsive subset of NK cells are those that express multiple inhibitory receptors that can be engaged by neighboring cells by means of their self-MHC class I molecules. This cardinal "rheostat concept" of NK cell licensing has been primarily demonstrated using gene knockouts and transgenic mice

Related Article: p. 4781

Conflict of interest: Laurence Zitvogel has received research support from Lytix Biopharma and Transgene and consultancy fees from GlaxoSmithKline and Ono Pharmaceuticals. Laurence Zitvogel is on the scientific advisory board of Immune Design, Bristol-Myers Squibb, and Transgene. Guido Kroemer has received consultancy fees and research support from Bayer HealthCare and has also received research support from GlaxoSmithKline and Lytix Biopharma. Reference information: / Clin Invest. 2014;124(11):4687-4689. doi:10.1172/JCI78531.

under steady-state conditions. It has been an open conundrum whether this concept would also apply to neoplastic diseases in which cancer cells (through genetic or epigenetic alterations) often manifest with a loss of MHC class I molecule expression, perhaps under the selective pressure of tumor antigen-reactive $\mathrm{T}$ lymphocytes (3).

\section{MHC class I-deficient tumor cells induce NK cell anergy}

In this issue of the JCI, Ardolino et al. (4) reveal that NK cell anergy is induced in WT mice bearing RMA-S lymphomas, which lack antigen peptide transporter 2 (Tap2) and are thereby MHC class I deficient. The anergic state of NK cells manifested as hyporesponsiveness of intratumoral NK cells ex vivo following restimulation with either plate-bound antibodies against the NK cell-activating receptors NKR-P1C or NKp46 or with YAC-1, an MHC-mismatched tumor cell line. In these conditions, RMA-S lymphoma-infiltrating NK cells failed to degranulate and release IFN $-\gamma$. NK cell anergy was an early and local phenomenon, occurring as early as seven days after tumor inoculation and only within the cancer infiltrate and in the tumor-draining lymph node. Moreover, NK cell anergy could be interpreted as a "dominant-negative" phenotype, as it was observed even in conditions in which only a minority of the tumor cells had lost MHC class I molecules (4)

Defective effector functions of RMA-S lymphoma-infiltrating NK cells could be directly ascribed to the loss of MHC class I expression in growing tumors, as RMA-S tumors in which MHC class I expression was restored via the reintroduction of Tap2 did not contain anergic NK cells. Moreover, removal of MHC class I expression by knocking out the $\beta 2$ microglobulin or Tap2 genes in myeloid leukemia-derived C1498 cells promoted the 


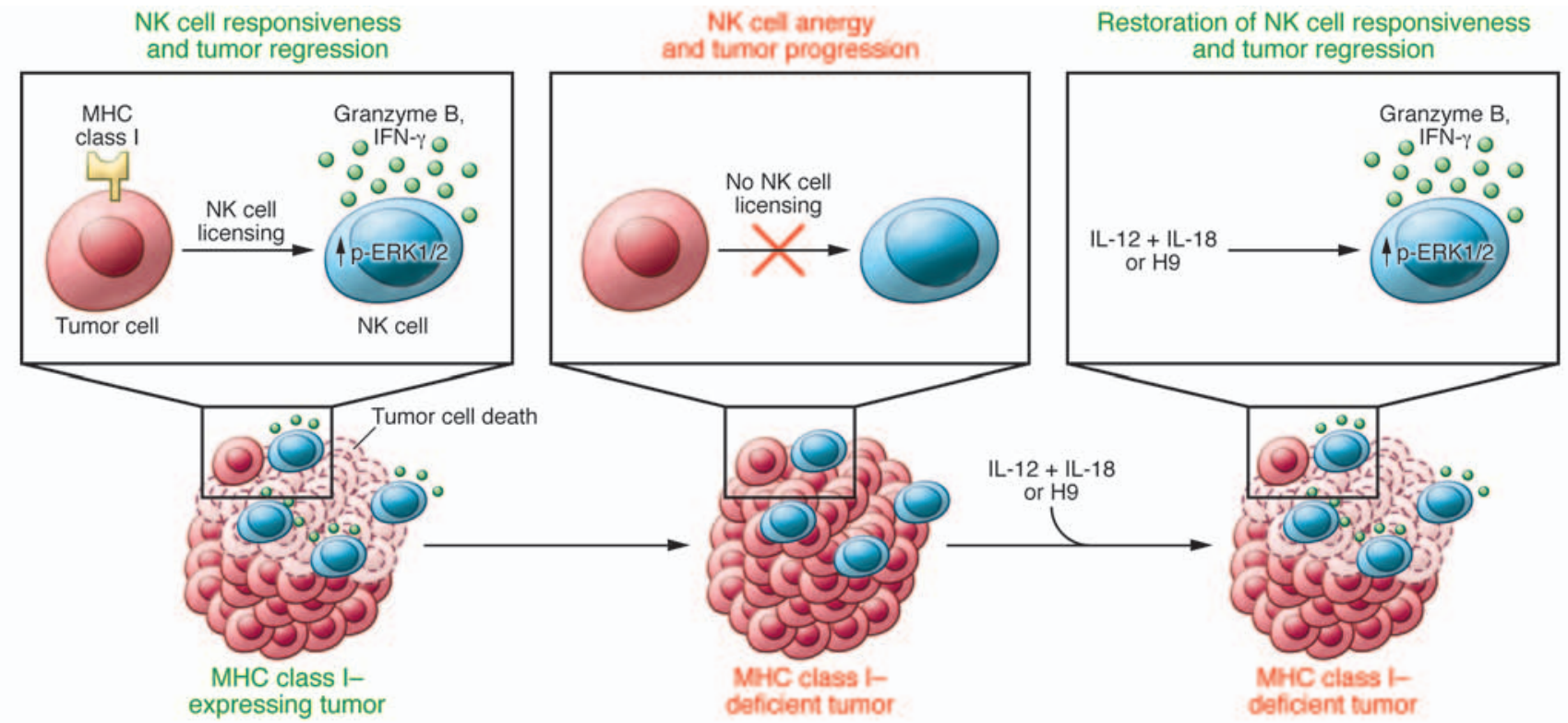

Figure 1. Restoring intratumoral NK cell functions with cytokines. When tumor cells lose MHC class I expression, which can occur for a variety of reasons, they disarm the function of NK cells, which become "unlicensed." However, upon administration of the combination of IL-12 plus IL-18 or H9, NK cells regain their functions, including phosphorylation of the stress kinase ERK1/2, production of granzyme B, and secretion of IFN- $\gamma$, and recover their capacity to control tumor progression.

generation of tumors that were infiltrated by anergic NK lymphocytes, while MHC class I-sufficient WT C1498 tumors contained functional NK cells. Of note, the functional defect of NK cells residing in MHC class I-deficient tumors could not be ascribed to variegated surface expression of NK cell inhibitory receptors (4). The expression of MHC class I molecules failed to influence NK cell trafficking or proliferation in situ, as MHC class I-deficient or -proficient tumors contained similar proportions of tumor-residing NK cells. Finally, the NK cell anergy associated with MHC class I-deficient tumors was not due to an increase in production of immunosuppressive cytokines, such as TGF- $\beta$ or IL-10, Tregs, or myeloid-derived suppressor cells (Figure 1 ).

Intratumoral NK cell hyporesponsiveness was associated with a dampening of early activation signals, specifically those that mediate phosphorylation of ERK1/2 (4), a mechanism previously described in NK cells differentiating in MHC class I-deficient mice or in WT NK cells invading MHC class I-proficient tumors (2). Altogether, these findings indicate that NK cells are progressively unlicensed as a consequence of the sustained loss of MHC class I molecules on cancer cells.

\section{Cytokines restore function in anergic NK cells}

Importantly, although NK cells derived from $\beta 2$ microglobulin-deficient mice or MHC class I-deficient tumors were refractory to stimulation via cell surface-activating receptors, they were able to respond to specific cytokines, such as the combination of IL-12 plus IL-18 or H9, which is an artificial cytokine engineered to stimulate the $\beta / \gamma$ IL-2 receptor heterodimer but not the $\alpha / \beta / \gamma$ IL-2 receptor complex (ref. 5 and Figure 1). Such exogenous stimuli (IL-12 plus IL-18 or H9) could restore ERK1/2 phosphorylation, IFN- $\gamma$, secretion, as well as the functional capacity of NK cells to eradicate MHC class I-deficient tumors. In contrast, the growth of MHC class Isufficient tumors was not affected by the administration of these cytokines. In this context, it is important to note that it has previously been shown that lack of NK cell licensing can be reversed by exposing NK cells to IL-12 (6) or by systemic infection of mice with Listeria or herpes viruses (7).

\section{Clinical implications}

The findings described by Ardolino et al. (4) offer a number of clinical prospects. In most human tumors, NK cells exhibit defective effector functions $(8,9)$. Constitutive or therapy-induced NK cell effector functions are likely to affect the clinical course of several human malignancies, including leukemia, multiple myeloma, neuroblastoma, gastrointestinal sarcoma, and kidney and lung carcinoma $(10,11)$.

Loss or downregulation of MHC class I expression has been widely reported in cancers that result from oncogene activation, such as those with mutations in NMYC, c-MYC, HER2/neu, or BRAF, and in cancers associated with inhibition of the $\mathrm{NF} / \mathrm{\kappa B}$ pathway $(12,13)$. Moreover, MHC class I loss variants become more frequent in the tumor cell population after stimulation with tumor-specific $\mathrm{T}$ cells by therapeutic vaccination (14) or treatment with imatinib mesylate (15), presumably as a result of $\mathrm{T}$ cell-mediated immunoselection. Incidentally, it is conceivable that blocking the NK cell inhibitory receptors (for instance with lirilumab [IPH2101]) might result in a similar paradoxical inhibition of NK cell functions, as observed in the presence of MHC class I-negative target cells (16).

Combating NK cell anergy by systemic or local delivery of inflammatory signals, such as with activated dendritic cells or cytokines, could constitute a valuable therapeutic option (5). Agents that stimulate IL- $2 \mathrm{R} \beta / \gamma$ signaling without preferential binding to the high-affinity IL- $2 \mathrm{R} \alpha / \beta / \gamma$ complex, which is constitutively expressed on 
Treg cells, may effectively boost NK cells without stimulating unwarranted immunosuppressive circuits $(16,17)$. Multiple approaches that use IL-12 have been or are being evaluated in advanced cancers, alone or together with conventional chemotherapy or mAbs targeting growth factors. IL-12 can be administered as a recombinant protein or via plasmid electroporation as well as in replication-incompetent adenoviral vectors. IL-12 is also being developed in the context of adoptive cell transfer immunotherapy with genetically engineered tumor-infiltrating lymphocytes (18). Results from recent phase I/II trials evaluating the safety and efficacy of recombinant IL-18 (SB-485232; GlaxoSmithKline) in stage IV melanoma patients (19), alone or in combination with pegylated liposomal doxorubicin against ovarian cancers (20), suggest that IL-18 has limited anticancer activity as a single agent. However, pilot studies combining IL-12 and IL-18 have not yet been attempted in humans.

To our knowledge, the stratification of patient cohorts based on tumor MHC class I expression has so far not been considered in the context of immunotherapy. Given that reliable immunohistochemical methods for the detection of MHC class I molecules on cancer cells and CD3, CD8, FOXP3, or NKp46 on tumor-infiltrating lymphocytes are available $(13,15)$, it should be possible to monitor these parameters before and after administration of NK cell-stimulatory compounds or antibodies against tumor antigens that elicit potentially NK-mediated cytotoxic responses (21). We anticipate that NK immunotherapies will yield predictive biomarkers that will have an impact on the clinical management of cancer patients.

\section{Acknowledgments}

L. Zitvogel and G. Kroemer are supported by the Equipes labelisées par la Ligue contre le Cancer; the Agence Nationale de la
Recherche (ANR); the Association pour la Recherche sur le Cancer (ARC); Cancéropôle Ile-de-France; the Institut National du Cancer (INCa); the Fondation Bettencourt-Schueller; the Fondation de France; the Fondation pour la Recherche Médicale (FRM); the European Commission (ArtForce); the European Research Council (ERC); LabEx Immuno-Oncology; SIRIC Stratified Oncology Cell DNA Repair and Tumor Immune Elimination (SOCRATE); SIRIC Cancer Research and Personalized Medicine (CARPEM); and the Paris Alliance of Cancer Research Institutes (PACRI).

Address correspondence to: Laurence Zitvogel, INSERM U1015, CICBT507, University Paris XI, Institut Gustave Roussy, 114 rue Edouard Vaillant, Villejuif, 94805, France. Phone: 33.1.42.11.50.05; E-mail: laurence.zitvogel@gustaveroussy.fr.

1. Vivier E, Raulet DH, Moretta A, Caligiuri MA, Zitvogel L. Innate or adaptive immunity? The example of natural killer cells. Science. 2011;331(6013):44-49.

2. Narni-Mancinelli E, Ugolini S, Vivier E. Tuning the threshold of natural killer cell responses. Curr Opin Immunol. 2013;25(1):53-58.

3. Kärre K, Ljunggren HG, Piontek G, Kiessling R. Selective rejection of $\mathrm{H}$-2-deficient lymphoma variants suggests alternative immune defence strategy. Nature. 1986;319(6055):675-678.

4. Ardolino M, et al. Cytokine therapy reverses NK cell anergy in MHC-deficient tumors. J Clin Invest. 2014;124(11):4781-4794.

5. Levin AM, et al. Exploiting a natural conformational switch to engineer an interleukin-2 'superkine'. Nature. 2012;484(7395):529-533.

6. Grufman P, Kärre K. Innate and adaptive immunity to tumors: IL-12 is required for optimal responses. Eur JImmunol. 2000;30(4):1088-1093.

7. Sun JC, Lanier LL. Cutting edge: viral infection breaks NK cell tolerance to "missing self". JImmunol. 2008;181(11):7453-7457.

8. Mamessier E, et al. Human breast cancer cells enhance self tolerance by promoting evasion from NK cell antitumor immunity. J Clin Invest.
2011;121(9):3609-3622.

9. Messaoudene M, et al. Mature cytotoxic CD56(bright)/CD16(+) natural killer cells can infiltrate lymph nodes adjacent to metastatic melanoma. Cancer Res. 2014;74(1):81-92.

10. Borg C, et al. Novel mode of action of c-kit tyrosine kinase inhibitors leading to NK cell-dependent antitumor effects. JClin Invest. 2004;114(3):379-388.

11. Remark R, et al. Characteristics and clinical impacts of the immune environments in colorectal and renal cell carcinoma lung metastases: influence of tumor origin. Clin Cancer Res. 2013;19(15):4079-4091.

12. Seliger B, Ritz U, Ferrone S. Molecular mechanisms of HLA class I antigen abnormalities following viral infection and transformation. Int J Cancer. 2006;118(1):129-138.

13. Garrido F, Algarra I. MHC antigens and tumor escape from immune surveillance. Adv Cancer Res. 2001;83:117-158.

14. Jäger $\mathrm{E}$, et al. Immunoselection in vivo: independent loss of MHC class I and melanocyte differentiation antigen expression in metastatic melanoma. Int J Cancer. 1997;71(2):142-147.

15. Rusakiewicz $S$, et al. Immune infiltrates are prognostic factors in localized gastrointestinal stromal tumors. Cancer Res. 2013;73(12):3499-3510.

16. Romagné $\mathrm{F}$, et al. Preclinical characterization of 1-7F9, a novel human anti-KIR receptor therapeutic antibody that augments natural killer-mediated killing of tumor cells. Blood. 2009;114(13):2667-2677.

17. Senovilla L, et al. Trial watch: prognostic and predictive value of the immune infiltrate in cancer. Oncoimmunology. 2012;1(8):1323-1343.

18. Vacchelli E, et al. Trial Watch: immunostimulatory cytokines. Oncoimmunology. 2012;1(4):493-506.

19. Tarhini AA, et al. A phase 2, randomized study of SB-485232, rhIL-18, in patients with previously untreated metastatic melanoma. Cancer. 2009;115(4):859-868.

20. Simpkins F, et al. Chemoimmunotherapy using pegylated liposomal Doxorubicin and interleukin-18 in recurrent ovarian cancer: a phase I dose-escalation study. Cancer Immunol Res. 2013;1(3):168-178.

21. Kellner C, Gramatzki M, Peipp M. Promoting natural killer cell functions by recombinant immunoligands mimicking an induced self phenotype. Oncoimmunology. 2013;2(6):e24481. 